\title{
Understanding biases when fitting disk truncations
}

\author{
Nicolás Cardiel ${ }^{1,3}$, Raffaella A. Marino ${ }^{2}$, Sergio Pascual ${ }^{1}$, M. Teresa \\ Ceballos $^{3}$, Armando Gil de Paz ${ }^{1}$ and Sebastián F. Sánchez ${ }^{4}$ \\ ${ }^{1}$ Depto. de Astrofísica y CC. de la Atmósfera, Universidad Complutense de Madrid, Spain \\ email: cardiel@ucm.es \\ ${ }^{2}$ Department of Physics, Institute for Astronomy, ETH Zürich, Switzerland \\ ${ }^{3}$ Instituto de Física de Cantabria (CSIC-Universidad de Cantabria), Santander, Spain \\ ${ }^{4}$ Instituto de Astronomía, Universidad Nacional Autónoma de México, México D.F.
}

\begin{abstract}
Truncations in the stellar population at the edges of disk galaxies are thought to be a common morphological feature (e.g., Erwin et al. 2005; and more recently Marino et al. 2016). In fact, using imaging data from the SDSS, Pohlen \& Trujillo (2006) showed that only $\sim 10 \%$ of face-on to intermediate inclined, nearby, late-type (Sb-Sdm) spiral galaxies have a normal/standard purely exponential disk down to the noise limit. In situations like these, the simultaneous fit of two lines, joined or not at an intermediate point (the break radius), constitutes a natural step towards the modelling of radial variation in surface brightness, metallicity, or any other relevant parameter. This work shows the results of simple simulations in which the simultaneous fit to two joined lines is compared to the simultaneous fit of two independent lines (i.e., two lines that do not necessarily coincide at an intermediate point), and also to the traditional single ordinary least squares fit. These simulations reveal some biases that should be taken into account when facing these kind of fitting procedures.
\end{abstract}

Keywords. methods: data analysis, methods: statistical, galaxies: general

In a previous work by Cardiel et al. (2016), and in order to simplify the initial analysis, numerical simulations of simultaneous fits to two lines were performed by assuming that the break radii could only be located at the mid point between adjacent data points. In this work this assumption has been relaxed, and the simulations indicate that, when fitting noisy data, the break radii tend to cluster around the abscissae corresponding to the fitted data sets. It is clear that the only way to confront fit biases like this is to compare the results of particular fits with simulations of analogous data sets.

The fitting method here described was used by Cardiel et al. (1998) to determine the break radius where star formation was taking place in central dominant galaxies, and more recently, Marino et al. (2016) have employed it within the CALIFA survey to analyse the possible connection between the external flattening observed in the ionisedgas metallicity gradients and the presence of breaks in the surface brightness profiles of disk galaxies.

\section{References}

Cardiel, N., Gorgas, J., \& Aragón-Salamanca, A. 1998, MNRAS 298, 977

Cardiel, N., Marino, R. A., Pascual, S., Ceballos M. T., Gil de Paz, A., \& Sánchez, S. F. 2016, $A S P-C S$ in press

Erwin, P., Beckman, J. E., \& Pohlen, M. 2005, ApJ (Letters) 626, L81

Marino, R. A., Gil de Paz, A., Sánchez, S. F., et al. 2016, A $\mathscr{3} A$ 585, id.A47

Pohlen, M. \& Trujillo, I. 2006, A\&A 454, 759 